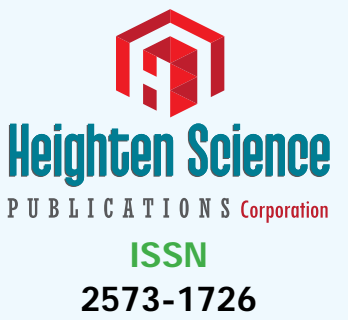

*Address for Correspondence: Dr. Gareth J ones, Centre for Pain Research, School of Clinical and Applied Sciences, Leeds Beckett University City Campus, Leeds LS1 3HE, United Kingdom, Email: g.j.jones@leedsbeckett.ac.uk

Submitted: 15 J une 2017

Approved: 13 J uly 2017

Published: 14 J uly 2017

Copyright: @2017 J ones G, et al. This is an open access article distributed under the Creative Commons Attribution License, which permits unrestricted use, distribution, and reproduction in any medium, provided the original work is properly cited.
Short Communication

\section{Characterisation of Delayed Onset of Muscle Soreness (DOMS) in the hand, wrist and forearm using a finger dynamometer: A pilot study}

\author{
Gareth J ones ${ }^{1 *}$, Kristina Grancharska² and Mark I J ohnson ${ }^{1}$ \\ ${ }^{1}$ Centre for Pain Research, School of Clinical and Applied Sciences, Leeds Beckett University, \\ United Kingdom \\ 'Faculty of Public Health and Sports, South-West University "Neofit Rilski", Blagoevgrad, \\ Bulgaria
}

\section{ABSTRACT}

Background: Experimentally-induced delayed-onset muscle soreness of large muscle groups is frequently used in as an injurious model of muscle pain. We wanted to develop an experimental model of DOMS to to mimic overuse injuries from sports where repeated finger flexion activity is vital such as rock climbing. The aim of this pilot study was to evaluate the utility of a 'finger trigger device' to induce DOMS in the fingers, hands, wrists and lower arms.

Methods: A convenient sample of six participants completed an experiment in which they undertook finger exercises to exhaustion after which measurements of pain, skin sensitivity to fine touch, forearm circumference and grip strength in the hand, wrist and forearm were taken from the experimental and contralateral nonexercised (control) arms.

Results: Pain intensity was greater in the experimental arm at rest and on movement when compared with the control arm up to 24 hours after exercise, although the location of pain varied between participants. Pressure pain threshold was significantly lower in the experimental arm compared with the control arm immediately after exercises locations close to the medial epicondyle but not at other locations. There were no statistical significant differences between affected and non-affected limbs for mechanical detection threshold, forearm circumference or grip strength.

Conclusion: Repetitive finger flexion exercises of the index finger by pulling a trigger against a resistance can induced DOMS. We are currently undertaking a more detailed characterization of sensory and motor changes following repetitive finger flexion activity using a larger sample.

\section{INTRODUCTION}

Delayed onset of muscle soreness (DOMS) is an acute myogenic condition classified as a type I muscle strain that normally occurs after strenuous eccentric and concentric exercise or after physical activity that is not accustomed for the individual [1]. Delayed onset of muscle soreness is related to microtrauma in sarcomeres and an associated inflammatory response with the involvement of neurotrophic factors manifesting as pain, stiffness, muscle tenderness, decreased strength and swelling $[2,3]$. Symptoms are delayed in onset with severity reaching a peak between 24 to 72 hours and disappearing by seven days after exercise. Experimentally-induced DOMS is frequently used in experimental settings as an injurious model of muscle pain $[4,5]$. Commonly, large muscle groups such as the hamstrings are used because protocols to induce DOMS are readily available. Disadvantages of using large muscle groups include discomfort and disability of body parts that impacts negatively on functions of daily living. We are interested in injuries associated with chronic overuse of fingers, hands, 
wrists and lower arms resulting from rock climbing [6-8]. Eccentric contractions of the wrist extensor muscles have been used in experimental studies to induce DOMS in the forearm $[9,10]$, but to our knowledge there have been no attempts to develop a model of DOMS in smaller muscle groups of hands. The aim of this pilot study was to evaluate the utility of a 'finger trigger device' to induce DOMS in the fingers, hands, wrists and lower arms. This was achieved by characterizing sensory and motor changes for 48 hours after exercise using the finger trigger device.

\section{METHODS}

\section{Study design}

A pre-post experimental design was used to evaluate the effect of finger exercises to exhaustion on pain, skin sensitivity to fine touch, forearm circumference and grip strength in the hand, wrist and forearm compared with participant's contralateral non-exercised hand, wrist and forearm.

\section{Participants}

A convenient sample of six participants was chosen for this pilot study. The study was approved by the Research Ethics Committee at the Leeds Beckett University, UK. Participants were recruited by announcing the experiment in lectures in our University. Interested volunteers received a participant information pack and were contacted again at least 48 hours later to be formally invited to take part in the study. Six, healthy, pain-free adults agreed to take part in, and completed all parts of the study (mean + SD age $=38.3+8.7$ years, 3 women, 3 men).

\section{Experimental procedure}

Experiments were conducted in the Pain Research Laboratory at our university and facilitated by one of our investigators (KG). During the study visit volunteers were briefed about the experiment and screened against the following exclusion criteria: a pre-existing medical condition; were currently seeking medical care; were taking medication; had experienced pain in the previous 6 months; had previously been diagnosed with a chronic pain condition; were experiencing disturbances in skin sensation such as sensitivity, numbness, or tingling; had a dermatological (skin) condition such as dermatitis, eczema or bacterial and fungal infections; were pregnant; regularly undertake vigorous exercise such as competitive sport. Eligible volunteers were formally invited to take part in the study and provided written consent.

Anthropometric data was taken and then measurements of self-reported pain, pressure pain threshold, forearm circumference and grip strength were recorded from the experimental (exercises) and control (no exercises) arms. Then each participant undertook a series of exercises with a view to inducing DOMS. A 'finger trigger device' designed by the authors was used to deliver exercises (Figure 1). The participants squeezed the trigger of the device using their index finger in time with a metronome (flexion=1s, extension=2s) until exhaustion they 'give-up' or for a maximum of 15 minutes. This was repeated following a 30 second rest. Measurements of self-reported pain, pressure pain threshold, forearm circumference and grip strength were taken from the experimental and control arms, immediately after exercises and then after 24 and 48 hours.

\section{Measurements}

Self-reported pain location, quality and intensity (VAS) at rest and on movement using the short form McGill questionnaire, that included a $100 \mathrm{~mm}$ visual analogue scale (VAS) $0=$ no pain and $100=$ worst pain imaginable) to measure present pain intensity.

Pressure pain threshold using a pressure algometer probe of $1 \mathrm{~cm}^{2}$, rate of pressure $=50 \mathrm{kPas}^{-1}$ (SenseLab Algometer, SOMEDIC, Sweden). For discussion regarding the validity and reliability of the measurement tool refer to [11]. 
Pressure pain algometry was applied at the following places on the anterior forearm and hand (Figure 2):

P1 - $1 \mathrm{~cm}$ above medial epicondyle of the humerus

P2 - $1 \mathrm{~cm}$ under medial epicondyle of the humerus

P3 - Muscle belly lateral

P4 - Muscle belly medial

P5 - Muscle tendons (fingers flexors)-middle of forearm

P6 - $1 \mathrm{~cm}$ above the radial styloid process-middle of forearm

Mechanical detection threshold using monofilaments (SenseLab Aesthesiometer, SOMEDIC, Sweden) applied at right angles to skin overlying the belly of flexor digitorum profundus muscle using a method of descending limits until the touch from one of the monofilaments could not be detected. The handle of the monofilament was
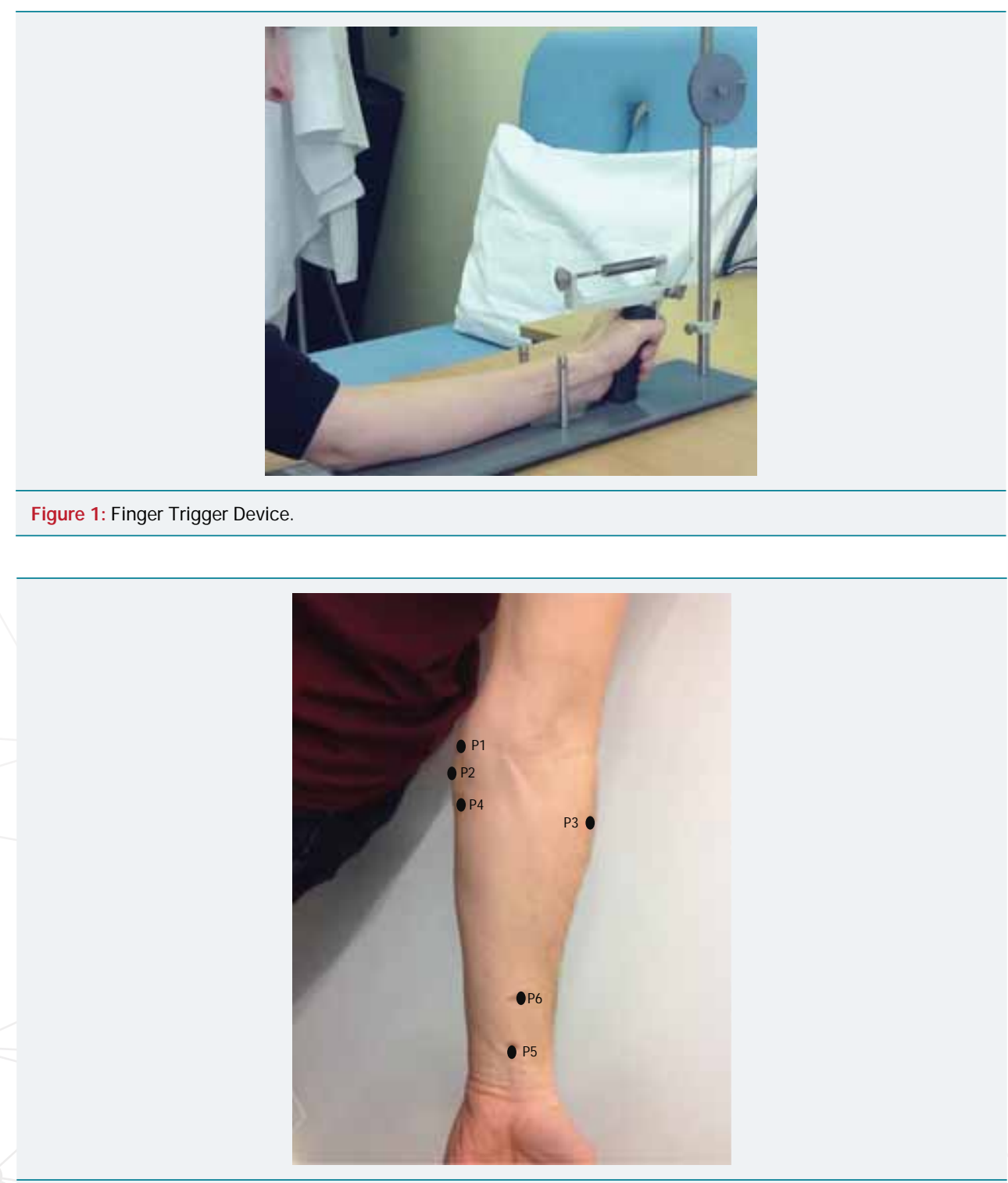

Figure 2: Location of measurements for pressure pain threshold and mechanical detection threshold. P1 = Point $1-1 \mathrm{~cm}$ above medial epicondyle; P2 = Point 2-1 cm under medial epicondyle; P3 = Point 3 - Muscle belly lateral lateral aspect of forearm in line with Point 4; P4 =Point $4-$ Muscle belly medial $-6 \mathrm{~cm}$ below medial epicondyle; P5 $=$ Point 5 - Muscle tendons (fingers flexors) at middle of the forearm $4 \mathrm{~cm}$ above Point $6 ;$ Point $6-1 \mathrm{~cm}$ above radial styloid process at middle of forearm. 
held parallel to the skin and pressure applied so that the filament buckled. The force applied by the thinnest monofilament that the participant could detect was recorded. For discussion regarding sensory threshold testing using nylon monofilaments refer to [12].

Forearm circumference (girth) was measured $6 \mathrm{~cm}$ inferior medial epicondyle with the arm held parallel to the ground with the palm facing upwards (supination) using a measuring tape.

Maximal grip strength was measured using a hand dynamometer (JAMAR ${ }^{\circledR}$ Hydraulic Hand Dynamometer, Patterson Medical, UK) with the participant standing and the forearm parallel to the ground at 90 degrees of flexion at the elbow and the hand in a neutral position. Participants were instructed to grip and squeeze the device as fast and as hard as they could for 3-5 seconds. Three measurements were taken alternately from each hand starting with the left hand. For discussion regarding the test-retest reliability of the measurement tool refer to [13].

Data analysis

Differences between experimental and control arms were calculated for each participant at each time point. All data was normally distributed and analyzed using paired t-tests (mean+SD) with alpha set at 0.05 .

\section{RESULTS}

\section{Induction of DOMS}

One participant did not 'give-up' (i.e. reach exhaustion) within the 15-minute timelimit for the finger exercises on run one or two. The mean+SD time to exhaustion for the other five participants was $178.0+109.5 \mathrm{~s}$ for run one and $87.8+39.7 \mathrm{~s}$ for run two $(n=5)$.

\section{Self-reported pain}

There was no pain at baseline reported by any of the six participants. All participants reported pain in the experimental arm at rest and on movement (i.e. finger flexion with and without resistance) immediately after exercises to induce DOMS and there were statistically significant differences between experimental and control arms (Table 1). At 24 hours three participants reported pain in the experimental arm and at 48 hours five participants reported pain on finger flexion without resistance in the experimental arm but there were no statistically significant differences between experimental and control arms. All participants reported pain in the experimental arm during finger flexion with resistance at 24 and 48 hours although there were only statistically significant differences between experimental and control arms at the 24 hour time point. The location of pain varied between participants and included the index finder, thumb, palm, wrist, middle of forearm, upper arm (m.biceps brachii) and shoulder. Aching and heavy were the most common pain descriptors.

Pressure pain threshold was significantly lower in the experimental arm compared with the control arm immediately after exercises at two of the six locations: $1 \mathrm{~cm}$ above the medial epicondyle and $1 \mathrm{~cm}$ under the medial epicondyle. There were no other statistically significant differences for locations or time points. Interestingly, many participants commented that they felt that the experimental arm was more sensitive to the pressure stimuli in the experimental arm at most locations at 24 hours and 48 hours although this was not reflected in measurements.

There were no statistical significant differences between affected and non-affected limbs for mechanical detection threshold, forearm circumference or grip strength. 
Table 1: Mean $\pm S D$ study data. $P$ values indicate differences in measurements between Experimental and Control arms calculated using paired-tests (two-tailed). The locations of the points used to measure pressure pain threshold (P1P6) are provided in the Methodology section.

\begin{tabular}{|c|c|c|c|c|}
\hline Measurement & Baseline & Immediately After & 24 hours & 48 hours \\
\hline \multicolumn{5}{|l|}{ Self-Reported Pain at Rest } \\
\hline - Experimental arm & $0 \pm 0$ & $33.0 \pm 25.1$ & $13.8 \pm 24.1$ & $1.7 \pm 4.1$ \\
\hline - Control arm & $0 \pm 0$ & $0.5 \pm 1.2$ & $0 \pm 0$ & $0 \pm 0$ \\
\hline - Difference & $0 \pm 0$ & $-32.5 \pm 25.48$ & $-13.8 \pm 24.1$ & $1.7 \pm 4.1$ \\
\hline - $\quad P$ value & 1 & 0.026 & 0.22 & 0.36 \\
\hline \multicolumn{5}{|c|}{$\begin{array}{l}\text { Self-Reported Pain Finger Flexion } \\
\text { without Resistance }\end{array}$} \\
\hline - $\quad$ Experimental arm & $0 \pm 0$ & $31.3 \pm 16.0$ & $10.83 \pm 14.73$ & $0.83 \pm 1.6$ \\
\hline - Control arm & $0 \pm 0$ & $0.83 \pm 2.04$ & $0 \pm 0$ & $0.3 \pm 0.8$ \\
\hline - Difference & $0 \pm 0$ & $-30.5 \pm 17.01$ & $-10.83 \pm 14.73$ & $-0.5 \pm 1.97$ \\
\hline - $\quad$ P value & 1 & 0.007 & 0.13 & 0.56 \\
\hline \multicolumn{5}{|c|}{$\begin{array}{l}\text { Self-Reported Pain Finger Flexion with } \\
\text { Resistance }\end{array}$} \\
\hline - $\quad$ Experimental arm & $0.33 \pm 0.81$ & $44.3 \pm 22.2$ & $12.67 \pm 9.81$ & $14 \pm 18.1$ \\
\hline - Control arm & $0.33 \pm 0.81$ & $2.3 \pm 4.41$ & $0 \pm 0$ & $0.5 \pm 1.22$ \\
\hline - Difference & $0 \pm 0$ & $-42.0 \pm 24.75$ & $-12.67 \pm 9.81$ & $-13.5 \pm 18.43$ \\
\hline - $\quad P$ value & 1 & 0.008 & 0.025 & 0.13 \\
\hline \multicolumn{5}{|l|}{ Pressure Pain Threshold (KPa) } \\
\hline \multicolumn{5}{|l|}{ P1 } \\
\hline - $\quad$ Experimental arm & $335.5 \pm 125.8$ & $183 \pm 115.2$ & $237.2 \pm 148.4$ & $236 \pm 105.3$ \\
\hline - Control arm & $273 \pm 106.2$ & $334.5 \pm 96.5$ & $272.5 \pm 98.3$ & $260.3 \pm 134.1$ \\
\hline - Difference & $-62.5 \pm 62.47$ & $151.5 \pm 115.95$ & $35.3 \pm 116.92$ & $24.3 \pm 83.78$ \\
\hline - $\quad P$ value & 0.06 & 0.02 & 0.49 & 0.5 \\
\hline \multicolumn{5}{|l|}{$\mathrm{P} 2$} \\
\hline - $\quad$ Experimental arm & $259.5 \pm 117.0$ & $272.8 \pm 102.1$ & $239.8 \pm 99.7$ & $207.7 \pm 113.5$ \\
\hline - Control arm & $269.8 \pm 131.6$ & $286.0 \pm 118.8$ & $252.2 \pm 89.8$ & $213.8 \pm 107.7$ \\
\hline - $\quad$ Difference & $10.3 \pm 66.73$ & $13.2 \pm 34.33$ & $12.3 \pm 55.60$ & $6.2 \pm 84.50$ \\
\hline - $\quad$ P value & 0.72 & 0.39 & 0.61 & 0.86 \\
\hline \multicolumn{5}{|l|}{ P3 } \\
\hline - $\quad$ Experimental arm & $337.5 \pm 104.6$ & $318.0 \pm 116.3$ & $243.3 \pm 86.1$ & $273.5 \pm 117.1$ \\
\hline - Control arm & $325.8 \pm 158.9$ & $300.2 \pm 98.9$ & $289.5 \pm 104.0$ & $265.8 \pm 111.1$ \\
\hline - $\quad$ Difference & $-11.7 \pm 66.25$ & $-17.8 \pm 89.38$ & $46.2 \pm 87.05$ & $-7.7 \pm 66.95$ \\
\hline - $\quad$ P value & 0.68 & 0.64 & 0.25 & 0.79 \\
\hline \multicolumn{5}{|l|}{ P4 } \\
\hline - Experimental arm & $387.2 \pm 170.0$ & $336.5 \pm 132.2$ & $286.2 \pm 138.2$ & $313.0 \pm 134.2$ \\
\hline - Control arm & $386.5 \pm 157.7$ & $339.5 \pm 103.5$ & $308.5 \pm 123.7$ & $328.2 \pm 104.3$ \\
\hline - $\quad$ Difference & $-0.7 \pm 99.80$ & $3 \pm 86.82$ & $22.3 \pm 27.96$ & $15.2 \pm 36.87$ \\
\hline - $\quad$ P value & 0.99 & 0.93 & 0.11 & 0.36 \\
\hline \multicolumn{5}{|l|}{ P5 } \\
\hline - Experimental arm & $286 \pm 131.3982$ & $341.3 \pm 154.5$ & $303.2 \pm 128.8$ & $286.3 \pm 83.4$ \\
\hline - Control arm & $289.4 \pm 91.2$ & $335.5 \pm 136.2$ & $310.8 \pm 129.2$ & $320.2 \pm 127.5$ \\
\hline - Difference & $3.4 \pm 125.96$ & $-5.8 \pm 67.19$ & $7.7 \pm 13.35$ & $33.8 \pm 69.90$ \\
\hline - $\quad P$ value & 0.95 & 0.84 & 0.22 & 0.3 \\
\hline \multicolumn{5}{|l|}{ P6 } \\
\hline - Experimental arm & $331.4 \pm 147.5$ & $291.7 \pm 106.7$ & $280.0 \pm 150.5$ & $311.5 \pm 113.4$ \\
\hline - Control arm & $310.8 \pm 109.8$ & $320.8 \pm 89.6$ & $255.8 \pm 160.1$ & $292.8 \pm 81.8$ \\
\hline - $\quad$ Difference & $-20.6 \pm 43.61$ & $29.2 \pm 82.11$ & $-24.2 \pm 104.68$ & $-18.7 \pm 48.61$ \\
\hline - $\quad$ P value & 0.35 & 0.42 & 0.6 & 0.39 \\
\hline \multicolumn{5}{|c|}{$\begin{array}{l}\text { Mechanical Detection Threshold ( } \mathrm{g} / \\
\mathrm{mm}^{2} \text { ) }\end{array}$} \\
\hline - Experimental arm & $4 \pm 1.76$ & $4.7 \pm 6.1$ & $4.6 \pm 4.8$ & $3.7 \pm 2.5$ \\
\hline - Control arm & $3.23 \pm 2.07$ & $4.7 \pm 4.6$ & $4.18 \pm 1.72$ & $4.8 \pm 2.4$ \\
\hline - Difference & $-0.77 \pm 0.97$ & $-0.03 \pm 1.55$ & $-0.42 \pm 3.16$ & $1.13 \pm 2.0$ \\
\hline - $\quad P$ value & 0.11 & 0.96 & 0.76 & 0.22 \\
\hline \multicolumn{5}{|l|}{ Forearm Circumference $(\mathrm{cm})$} \\
\hline - Experimental arm & $28.3 \pm 3.3$ & $28.6 \pm 3.2$ & $28.5 \pm 3.2$ & $28.3 \pm 2.9$ \\
\hline - Control arm & 28.33 .3 & $28.4 \pm 3.25$ & $28.3 \pm 3.11$ & $28.23 \pm 3.0$ \\
\hline - Difference & $0.07 \pm 0.08$ & $-0.18 \pm 0.48$ & $-0.18 \pm 0.69$ & $-0.08 \pm 0.22$ \\
\hline - $\quad$ P value & 0.1 & 0.39 & 0.54 & 0.4 \\
\hline \multicolumn{5}{|l|}{ Maximal grip strength $(\mathrm{kg})$} \\
\hline - Experimental arm & $38.2 \pm 9.7$ & $36.9 \pm 12.0$ & $37.9 \pm 11.35$ & $37.4 \pm 11.3$ \\
\hline - Control arm & $38.4 \pm 9.4$ & $38.3 \pm 12.1$ & $37.8 \pm 12.4$ & $38.6 \pm 12.8$ \\
\hline - $\quad$ Difference & $0.13 \pm 3.6$ & $1.77 \pm 4.28$ & $-0.1 \pm 4.7$ & $1.18 \pm 3.44$ \\
\hline - $\quad$ P value & 0.93 & 0.47 & 0.96 & 0.44 \\
\hline
\end{tabular}




\section{DISCUSSION}

The findings of this pilot study demonstrate the utility of a finger trigger device to induce DOMS associated with eccentric exercises of a single finger. Our protocol used two periods of finger exercise to exhaustion separated by a 30 second rest. Only one participant did not reach exhaustion within the 15-minute time limit suggesting that this protocol was viable.

DOMS was characterised as pain at rest and on movement that was distributed in the fingers, hands, wrists and lower arms at rest and on movement and persisted up to 24 hours after exercise. Pain at rest in the experimental arm had diminished within 24 hours for three participants but persisted up to 48 hours during finger movement against a resistance in all participants. The characteristics and time course of DOMS was similar to that previously reported for other body parts including arms and legs [1]. The aching nature of the pain is likely to reflect musculoskeletal trauma [2,3], although the location of pain differed substantially between participants and included fingers, palm of hand, wrist, lower and upper arm and shoulder. This might reflect variations in strategies used to pull the finger trigger that lead to recruitment of different muscle groups.

We used blunt pressure algometry to ascertain whether post-exercise pain was driven by peripheral input from deep seated strictures such as muscle and connective tissue [4]. Sensitivity to blunt pressure was pronounced immediately after finger trigger exercises $1 \mathrm{~cm}$ above the medial epicondyle and $1 \mathrm{~cm}$ under the medial epicondyle but not at other locations. We were surprised that there were no significant reductions in pressure pain thresholds in the experimental at any other location or time point [14], although participants commented that they believed that they were more sensitive to pressure stimuli on the experimental arm. The failure to detect statistically significant differences may be due in part to between-participant variability in the distribution of pain increasing between-participant variance pressure pain thresholds at each location.

We measured mechanical detection threshold to ascertain whether post-exercise pain had a cutaneous component (tactile allodynia) [15]. There were no statistical significant differences between experimental and control arms. Thus, we have tentative evidence that DOMS associated with finger trigger exercises is driven by musculoskeletal rather than cutaneous structures.

There were no statistically significant changes in forearm circumference following exercise suggesting that there was no appreciable oedema. We were surprised that there were no statistically significant differences in grip strength between the experimental and control arms as the task involved the use of anatomical structures involved in finger trigger exercises [16]. Participants commented that they tended to guard the index finger and recruit non painful fingers during the grip strength test and this may explain our failure to detect differences between arms.

In conclusion, the findings of this study suggest that this model of DOMS has the potential to mimic overuse injuries from sports where repeated finger flexion activity is vital such as rock climbing. We plan to follow up this preliminary study with a more detailed characterization of sensory and motor changes following repetitive finger flexion activity using a larger sample.

\section{REFERENCES}

1. Dannecker EA, Koltyn KF. Pain during and within hours after exercise in healthy adults. Sports Med. 2014; 44: 921942. Ref.: https://goo.gl/eKgZnA

2. Lewis PB, Ruby D, Bush-J oseph CA. Muscle soreness and delayed-onset muscle soreness. Clin Sports Med. 2012; 31: 255-262. Ref.: https://goo.gl/4Mqspv 
3. Mizumura K, Taguchi T. Delayed onset muscle soreness: Involvement of neurotrophic factors.J Physiol Sci. 2016; 66: 43-52. Ref.: https://goo.gl/rMW2Si

4. Olesen AE, Andresen T, Staahl C, Drewes AM. Human experimental pain models for assessing the therapeutic efficacy of analgesic drugs. Pharmacol Rev. 2012; 64: 722-779. Ref.: https://goo.gl/BH6XYo

5. Staahl C, Drewes AM. Experimental human pain models: a review of standardised methods for preclinical testing of analgesics. Basic Clin Pharmacol Toxicol. 2004; 95: 97-111. Ref.: https://goo.gl/2vYkPY

6. J ones G, Asghar A, Llewellyn DJ . The epidemiology of rock-climbing injuries. BrJ Sports Med. 2008; 42: 773-778. Ref.: https://goo.gl/amFyko

7. J ones G, J ohnson MI. A Critical Review of the Incidence and Risk Factors for Finger Injuries in Rock Climbing. Curr Sports Med Rep. 2016; 15: 400-409. Ref.: https://goo.gl/uvQkXa

8. J ones G, Llewellyn D, J ohnson MI. Previous injury as a risk factor for reinjury in rock climbing: a secondary analysis of data from a retrospective cross-sectional cohort survey of active rock climbers. BMJ Open Sport Exerc Med. 2015; 1. Ref.: https://goo.gl/oBAlja

9. Delfa de la Morena J M, Samani A, Fernandez-Carnero J , Hansen EA, Madeleine P. Pressure pain mapping of the wrist extensors after repeated eccentric exercise at high intensity.J Strength Cond Res. 2013; 27: 3045-3052. Ref.: https://goo.gl/NV7G47

10. Madeleine $P$, Hansen EA, Samani A. Linear and nonlinear analyses of multi-channel mechanomyographic recordings reveal heterogeneous activation of wrist extensors in presence of delayed onset muscle soreness. Med Eng Phys. 2014; 36: 1656-1664. Ref.: https://goo.gl/BEp39s

11. Kinser AM, Sands WA, Stone MH. Reliability and validity of a pressure algometer.J Strength Cond Res. 2009; 23: 312-314. Ref.: https://goo.gl/S1Mwnv

12. Bove G. Mechanical sensory threshold testing using nylon monofilaments: The pain field's "Tin Standard". Pain. 2009; 124: 13-17. Ref.: https://goo.gl/unL6Zc

13. Bohannon RW, Schaubert KL. Test-retest reliability of grip-strength measures obtained over a 12-week interval from community-dwelling elders. J Hand Ther. 2005; 18: 426-427. Ref.: https://goo.gl/V9PJ mL

14. Black CD, Tynes BK, Gonglach AR, Waddell DE. Local and Generalized Endogenous Pain Modulation in Healthy Men: Effects of Exercise and Exercise-Induced Muscle Damage. Pain Med. 2016; 17: 2422-2433. Ref.: https://goo.gl/2gGKTF

15. Uddin Z, MacDermid J C. Quantitative Sensory Testing in Chronic Musculoskeletal Pain. Pain Med. 2016; 17. 16941703. Ref.: https://goo.gl/7bc4Nh

16. Khamwong P, Paungmali A, Pirunsan U, J oseph L. Prophylactic Effects of Sauna on Delayed-Onset Muscle Soreness of the Wrist Extensors. AsianJ Sports Med. 2015; 6. Ref.: https://goo.gl/mQZhzm 